\title{
DESIGN AND IMPLEMENTATION OF INTERFACE CIRCUITRY FOR CMOS-BASED SAW GAS SENSORS
}

\author{
F.M. Yasin, K.F. Tye, M.B.I. Reaz \\ Faculty of Engineering, Multimedia University \\ Cyberjaya, Selangor, Malaysia 63100
}

\begin{abstract}
The design and development of interface circuitries for CMOS-based SAW gas sensor is presented in this paper. The SAW sensor devices typically run at $R F$, requiring most design to have complex signal conditioning circuitry. Our approach attempt to design a simple architecture with reduced power consumption. The SAW sensors operate at $354 \mathrm{MHz}$. Simulation data show that the interface circuitries are ten times smaller with lower power supply, compare to existing work.
\end{abstract}

\section{INTRODUCTION}

Surface Wave Acoustic (SAW) gas sensors are small miniature sensors used to detect various gases and chemical compounds in the air. SAW gas sensors are currently being used to monitor hazardous chemical vapors, chemical warfare agents, potential fires and environmental pollutants. However, signals from the gas sensor needs to be processed before it can be converted into digital form for further processing. Hence signal processing circuitry is required in order to render the overall control system more efficiently. By incorporating the signal processing circuitry with the SAW gas sensor on the same CMOS substrate, a complete system on a chip (SoC) will be formed.

The development of miniaturized SAW gas sensors on one chip that are highly selective and sensitive have been going on for the past five years. However, the RF operating frequencies requires most designs to use complex signal conditioning circuitry. We attempt to produce a simpler architecture, by making comparison to existing architecture such as Hagleitner et. al[1].

This paper illustrates how each and every block of modules in the signal processing circuitry functions. The design techniques applied in order to optimize the performance of the circuits are also highlighted. Comparison with one signal processing circuitry is also made. This miniature gas sensing circuitry is believed to reduce the power consumption and the area of the chip.

\section{DESIGN}

\section{A. System Overview}

The gas sensor consists of four main blocks, namely the SAW gas sensor, frequency divider, frequency to voltage converter (FVC), analog multiplexer and analog to digital converter (ADC). The signal processing circuitry is able to detect the input, which is in the form of frequency ranging from $300 \mathrm{MHz}$ to $400 \mathrm{MHz}$, with a minimum sensitivity of approximately $10 \mathrm{MHz}$. The output is in the form of analog voltage in the range of $0.89 \mathrm{~V}$ to $2.06 \mathrm{~V}$.

\section{B. SAW Sensors}

This gas sensor consists of two SAW delay lines, where one delay line acts as the reference device while the other acts as the sensor, which is coated with a tungsten trioxide $\left(\mathrm{WO}_{3}\right)$ film as a sensing element for hydrogen sulfide $\left(\mathrm{H}_{2} \mathrm{~S}\right)$. The purpose of having dual delay lines is to compensate the environmental effects such as pressure variation and temperature drift. An algorithm has been developed to get the optimized solution for the SAW sensor's design parameters.

\section{Frequency Divider}

Frequency divider acts as prescaler, i.e. to divide the input frequency into lower frequency values. The six-NAND type frequency divider is chosen in this project. 


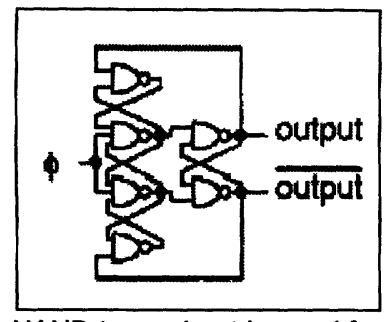

Figure 1 - Six-NAND type edge-triggered frequency divider

Figure 1 shows the design. Race conditions will not occur as the circuit is edge triggered. Only 34 transistors are used which saves area.

\section{Frequency to Voltage Converter}

The FVC is used to convert an input of frequency to an output of voltage. The FVC designed by $A$ Djemouai[2] is chosen for implementation because it consumes only a small area and it is able to provide outputs without ac ripples. These two conditions is important especially for the gas sensor signal processing circuitry where area and noise are some of the main concerns [3].

Figure 2 shows the schematic of the FVC designed. This FVC consists mainly of two equal values capacitors, a practical current source, a set of transistors as switches and a logic controller block (LCB). The practical current source provides current to drive the whole circuit. Basically there are three different phases of operation for this FVC. During these three different phases, different transistors are on, producing different operations. The switching of the transistors is controlled by the LCB.

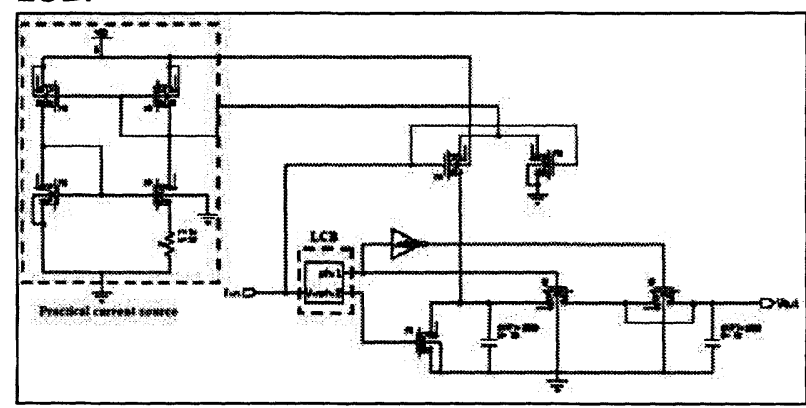

Figure 2 - FVC and its logic controller block

The practical current source designed here applies the bootstrap bias technique. The basic PMOS current mirror is selected as the current mirror in bootstrap configuration while the NMOS
Widlar current source serves as the current source counterpart.

The LCB design from Djemouai[2] is shown in Figure 3.

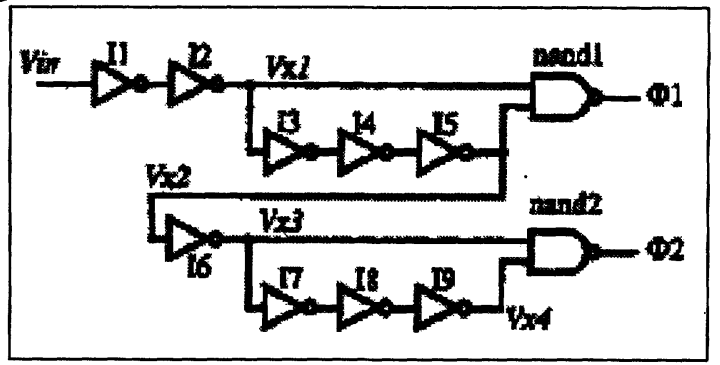

Figure 3 - LCB designed by A. Djemouai et al

The LCB circuit consists mainly of two NAND gates and a number of inverters. Inverters 11 and 12 are used to create a short delay in order to guarantee that the output signal phi1 will not rise up until after the input signal Vin reaches its logic high level. The produced signal $V \times 1$, is then inverted and delayed by a period of $T 1$, which corresponds to the delay of inverters 13 to 15 . The NAND gate, nand1, will then takes the signals $V \times 1$ and $V \times 2$ and produces the output signal phi1. Inverter I6 is included to make sure that the output signal phi2 will not rise up before the output signal phi1 reaches its logic low level. Vx3 generated from 16 is then inverted and delayed by a period of $\mathrm{T} 2$, corresponding to the delay of inverters 17 to 19 . The second NAND gate, nand2, will then take signals $V \times 3$ and $V \times 4$ to produce output signal phi2. Figure 4 shows the LCB control signal.

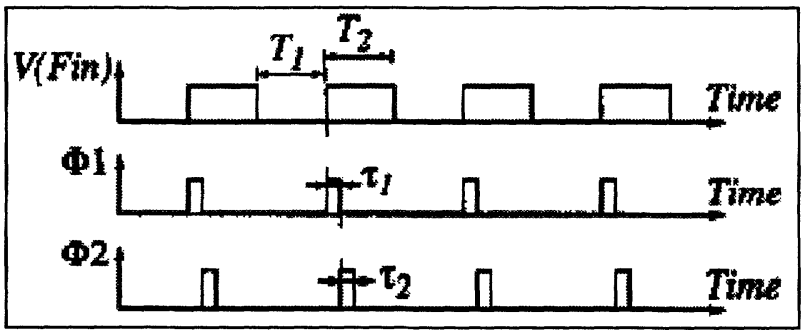

Figure 4 - LCB control signal

It was noticed that using the LCB circuit shown in Figure 3, the output signals phi1 and phi2 were wrong, as shown in Figure 5. Hence, modification was made by adding two inverters to the outputs of nand1 and nand2. The modified LCB was shown in Figure 6. 


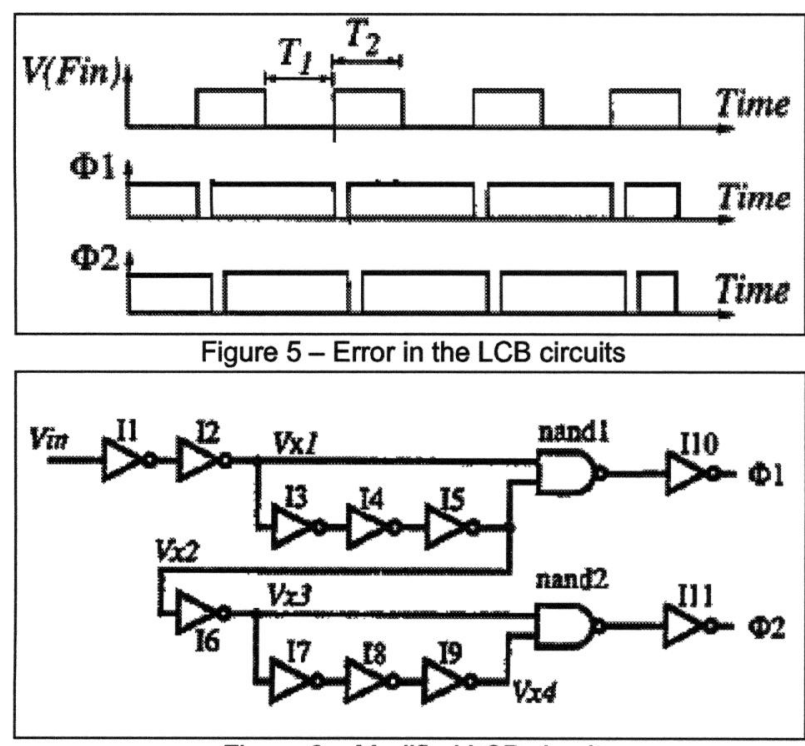

Figure 6 - Modified LCB circuit

With the modification, the LCB was able to produce the output as intended originally.

The current-starved inverter was used as delay cells. In current-starved inverter, limited current is used to discharge the load capacitance of the gate. When less current is supplied, the time needed to charge and discharge the load capacitance will increase. This will then provides a longer delay. Although this circuit requires more transistors, it provides more delay per cell, giving a much more better output signals compared to the CMOS inverter. Hence, less delay cells are required.

\section{E. Differential Amplifier}

A differential amplifier is used to amplify the difference (in the range of $\mathrm{mV}$ ) between two input signals coming from the sensors.

The differential amplifier using the basic twostage CMOS op amp is chosen for this project. This design has the less number of transistors, which Figure 7 shows the circuitry. The first stage of the op amp consists of a differential pair M1 and M2 driving a current mirror load, M3 and M4. The second stage consists of a common source amplifier, M5. The capacitor linking between the two stages is used for compensation purposes, i.e. to reduce the ripples at the output.

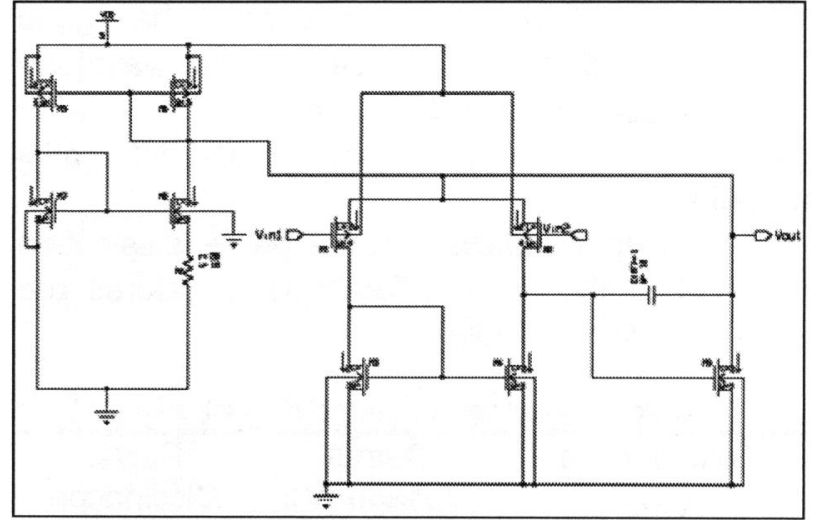

Figure 7 - Basic two-stage CMOS op amp

\section{RESULTS}

The CMOS SAW gas sensor is designed using Mentor Graphics design tools based on TSMC 0.35um technology. The frequency divider, frequency to voltage converter and the differential amplifier modules have been simulated successfully. The results obtained from the simulation have been compared with the analysis. The layout has been designed manually.

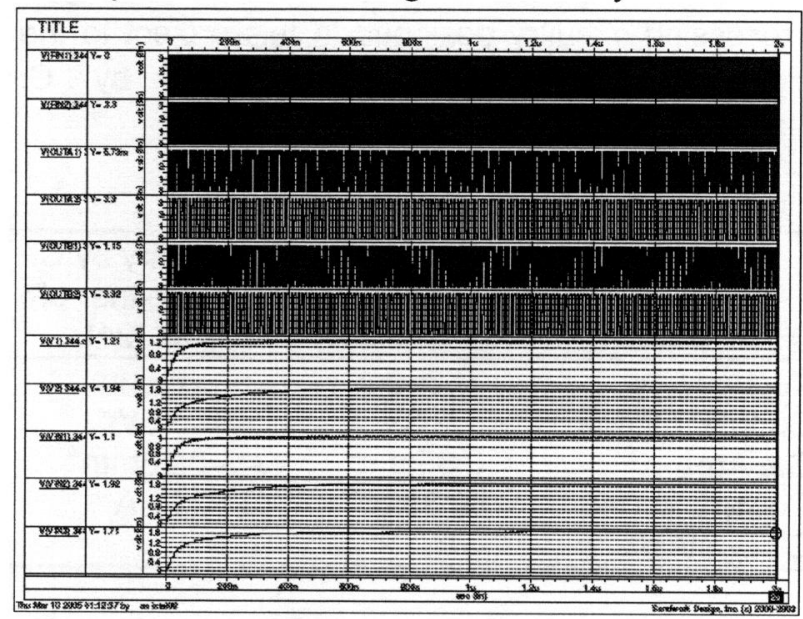

Figure 8 - Final output

Figure 8 illustrates the final output obtained from the signal processing circuitry designed in this project. The input frequencies, fin 1 is $344 \mathrm{MHz}$ while fin2 is set to be $354 \mathrm{MHz}$. Waveforms OUTA1 and OUTA2 are the outputs after going through the divide-by-2 frequency divider. Waveforms OUTB1 and OUTB2 are the outputs obtained after going through the divide-by-4 frequency divider. The outputs after passing through the FVC1 and FVC2 are labeled as V2 and V1. The signals labeling as 
VIN1 and VIN2 are the signals which had gone through the differential amplifier. The waveform which is labeled as VIN3 is the final output, after going through the frequency divider and differential amplifier.

The output voltage and the power dissipation obtained at the three different temperatures are each tabulated in Table 1.

Table 1: Output voltage and power dissipation

\begin{tabular}{ccc}
\hline $\begin{array}{c}\text { Temperature } \\
\left({ }^{\circ} \mathrm{C}\right)\end{array}$ & $\begin{array}{c}\text { Output } \\
\text { Voltage }(\mathrm{V})\end{array}$ & $\begin{array}{c}\text { Power } \\
\text { Dissipation } \\
(\mathrm{mW})\end{array}$ \\
\hline 25 & 1.71 & 38.35 \\
60 & 1.67 & 34.31 \\
85 & 1.67 & 31.77 \\
\hline
\end{tabular}

From the results of Table 1, one can observe that the output voltage is quite similar. This indicates that the circuit designed is not sensitive to temperature. The output waveform does not show significant ripples, indicating that the circuit has strong noise immunity, even at high frequencies.

Table 2 shows a comparison of the signal processing circuitry designed in this project to the signal processing circuitry designed by $C$. Hagleitner et al.

\begin{tabular}{lcc} 
& Table 2: Comparison \\
\hline & $\begin{array}{c}\text { Circuitry } \\
\text { designed in } \\
\text { this project }\end{array}$ & $\begin{array}{c}\text { Circuitry by C. } \\
\text { Hagleitner et } \\
\text { al. [21] }\end{array}$ \\
\hline Technology & $0.35 \mu \mathrm{m}$ & $0.5 \mu \mathrm{m}$ \\
Supply Voltage & $3.3 \mathrm{~V}$ & $5 \mathrm{~V}$ \\
Die Area & $0.85 \mathrm{~mm}^{2}$ & $8.48 \mathrm{~mm}^{2}$ \\
Power & $38.35 \mathrm{~mW}$ & $\mathrm{NA}$ \\
Dissipation & & \\
\hline
\end{tabular}

Our circuitry designed in this project is ten times smaller than the one designed by C.Hagleitner et al. In addition, it is able to operate at a lower supply voltage, i.e. 3.3V compared to $5 \mathrm{~V}$. However, the circuit by Hagleitner et. al was able to take multiple inputs, which is our work in progress. In addition, we also compare our design to Hassani et. al.[4]-[5]. We found that our design are simpler and yet produce comparable results with reference to other works.

\section{CONCLUSION}

A signal processing circuitries for CMOS SAW gas sensor is designed in this project. The final layout of the circuitry occupies an area of $0.85 \mathrm{~mm}^{2}$. The power dissipation at normal temperature $\left(25^{\circ} \mathrm{C}\right)$ is about $38.35 \mathrm{~mW}$. We also found that the circuit has strong noise immunity and temperature sensitivity. The overall block diagram of the SAW gas sensor is shown in Figure 10.

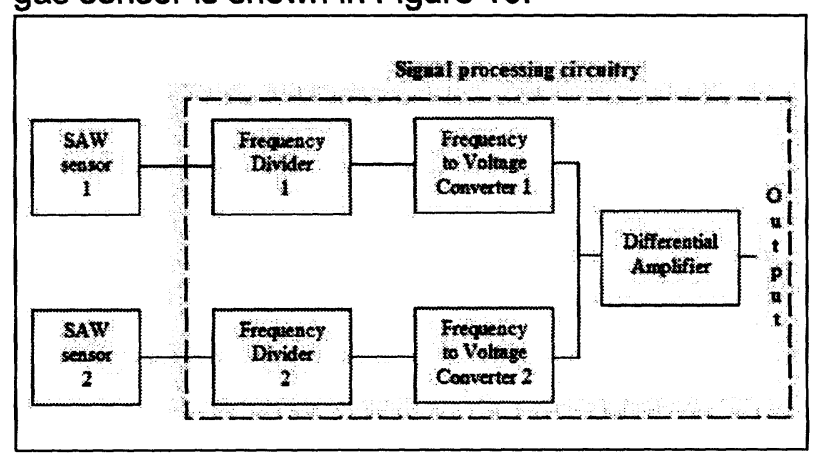

Figure 10: Overall block diagram

\section{REFERENCES}

1. C. Hagleitner, D. Barrettino, A. Hierlemann, O. Brand, H. Baltes, "CMOS Single-Chip Gas Detection System Comprising Capacitive, Calorimetric and Mass-Sensitive Microsensors", "IEEE Journal of Solid-State Circuits, Vol.37, No.12, Dec 2002.

2. A. Djemouai, M. Sawan and M. Slamani, "High Performance Integrated CMOS Frequency-toVoltage Converter", International Conference on Microelectronics, Tunisia, 63-66, Dec 1998.

3. Surakampontorn, W., Chonbodeechalermroong, Y., Bunjongjit, S., "An Analog Sinusoidal Frequency-to-Voltage Converter" the transactions of IEEE on Instrumentation and Measurement, Vol. 40, No. 6, December 1991, pg 925-929.

4. F. Hassani, O. Tigli, S. Ahmadi, C. Korman and M.E. Zaghloul "Integrated CMOS Surface Acoustic Wave Gas Sensor: Design and Characteristics", Proceedings of IEEE Sensors 2003, vol. 2, 2003.

5. S. Ahmadi, C. Korman, M. Zaghloul and K.H. Huang, " CMOS Integrated Gas Sensor Chip Using SAW Technology", Proceedings of ISCAS 2003, pp. 848-851, 2003. 